\title{
Can marine bacteria be recruited from freshwater sources and the air?
}

\author{
Jérôme Comte ${ }^{1,2}$, Eva S Lindström ${ }^{1}$, Alexander Eiler ${ }^{1}$ and Silke Langenheder ${ }^{1}$ \\ ${ }^{1}$ Department of Ecology and Genetics/Limnology, Uppsala University, Norbyvägen 18D, Uppsala, Sweden \\ and ${ }^{2}$ Centre d'Études Nordiques, UMI Takuvik, Départment de biologie-IBIS, Université Laval, Pavillon \\ Charles-Eugène-Marchand, 1030, avenue de la Médecine, Québec (Québec), Canada
}

\begin{abstract}
There is now clear evidence that microorganisms present biogeographic patterns, yet the processes that create and maintain them are still not well understood. In particular, the contribution of dispersal and its exact impact on local community composition is still unclear. For example, dispersing cells may not thrive in recipient environments, but may still remain part of the local species pool. Here, we experimentally tested if marine bacteria can be retrieved from freshwater communities (pelagic and sediment) and the atmosphere by exposing bacteria from three lakes, that differ in their proximity to the Norwegian Sea, to marine conditions. We found that the percentage of freshwater taxa decreased with increasing salinities, whereas marine taxa increased along the same gradient. Our results further showed that this increase was stronger for lake and sediment compared with air communities. Further, significant increases in the average niche breadth of taxa were found for all sources, and in particular lake water and sediment communities, at higher salinities. Our results therefore suggests that marine taxa can readily grow from freshwater sources, but that the response was likely driven by the growth of habitat generalists that are typically found in marine systems. Finally, there was a greater proportion of marine taxa found in communities originating from the lake closest to the Norwegian Sea. In summary, this study shows that the interplay between bacterial dispersal limitation and dispersal from internal and external sources may have an important role for community recovery in response to environmental change.
\end{abstract}

The ISME Journal (2014) 8, 2423-2430; doi:10.1038/ismej.2014.89; published online 6 June 2014

Subject Category: Microbial population and community ecology

\section{Introduction}

Recent progress in microbial biogeography has revealed patterns in community composition highly related to environmental gradients (Lozupone and Knight, 2007; Tamames et al., 2010). However, the underlying processes are not always clear. Although the role of dispersal and effects of environmental conditions in determining community composition have been reported in many studies (Hanson et al., 2012; Lindström and Langenheder, 2012), quantifying their specific contribution in the community assembly process represents one of the major challenges in biogeography research. The main difficulty is that both are acting simultaneously and their relative importance may depend on the level of observation, for example, habitat type, spatial and taxonomic scales (Hanson et al., 2012). A further complicating fact is that both dispersal and environmental filtering can have a historical

Correspondence: J Comte, Centre d'Études Nordiques, UMI Takuvik, Départment de biologie-IBIS, Université Laval, Pavillon Charles-Eugène-Marchand, 1030, avenue de la Médecine, Québec, Québec G1V 0A6, Canada.

E-mail: jerome.comte@takuvik.ulaval.ca

Received 13 November 2013; revised 18 April 2014; accepted 22 April 2014; published online 6 June 2014 dimension: As cells are dispersed to unsuitable environments where they can be stored and accumulate over time, they can later serve as inocula when conditions become favorable (Shade et al., 2012; Sjöstedt et al., 2012; Declerck et al., 2013; Gibbons et al., 2013).

The marine-freshwater transition is a good system to address the historical dimension of dispersal, as comparative studies have shown that saline and freshwater ecosystems generally show little overlap with respect to abundant bacterial taxa (Lozupone and Knight, 2007; Logares et al., 2009; Tamames et al., 2010; Newton et al., 2011). Nevertheless, marine bacteria should be found among the less abundant members of freshwater bacterial communities. Hence, it should be possible to retrieve typical marine bacteria (marine specialists) from freshwater communities when exposed to marine conditions.

There are different strategies by which freshwater bacterial communities can respond when exposed to a marine gradient. Compositional responses can result from the recruitment of taxa that present narrow niche breadth, that is, habitat specialists. Alternatively, opportunistic taxa with a wide niche breadth could be recruited, that is, habitat generalists. The extent to which habitat specialists or generalists 
are selected when bacterial communities are exposed to novel environments is largely unknown; however, there is evidence that both are important (Székely et al., 2013). Different recruitment sources can potentially be important, including external sources, such as atmospheric deposition (for example, Jones et al., 2008), that is, primarily precipitation, and internal sources, such as the water column and sediments. Marine taxa that enter the atmosphere during aerosol formation above oceans may, for example, be transported to freshwater ecosystems via air dispersal. On the other hand, it has been shown that air dispersal may also be a source for habitat generalists that are able to cope with a wide range of environmental conditions (Fahlgren et al., 2010). Other possible sources for the recruitment of marine bacteria to freshwater communities include bacteria within lake water and sediments, reflecting accumulation of cells that have been dispersed into the lake at some point during its history. Especially sediment communities should contain larger taxonomic pools for the recruitment of marine bacteria when compared with the water column, as they have a higher richness (for example, Lozupone and Knight, 2007), most likely a result of cell accumulation over time (Finlay et al., 1997; Hubert et al., 2009).

Here, we conducted an experiment to investigate the compositional response of three sources of bacterial communities, that is, lake water, sediments and air, obtained from three lakes, to the exposure to different degrees of marine conditions. First, we hypothesize that marine bacteria are present in freshwater communities and in the air species pool. Thus, our expectation is that we should retrieve marine taxa from these sources after exposure to marine conditions. Second, we hypothesize that marine bacteria are mainly present in sediments due to a greater accumulation of cells over time. Thirdly, we hypothesized that marine taxa should be retrieved to a greater extent from lakes situated closer to the sea. Fourthly, we hypothesized that the changes in community composition along the marine gradient should lead to a higher proportion of marine taxa in both lake water and sediments, whereas a higher proportion of habitat generalists should be retrieved from airborne communities.

\section{Materials and methods}

\section{Sampling sites and procedures}

To limit the potential effect of latitudinal gradients, we sampled three lakes of comparable latitude, but of different proximity to the sea. Therefore, we sampled in August 2010 (1) lake Lille Jonsvatn $\left(63^{\circ} 22^{\prime} \mathrm{N}, 10^{\circ}\right.$ $37^{\prime} \mathrm{E}$ ), which is located in central Norway, $20 \mathrm{~km}$ southeast from the city of Trondheim and the marine Trondheim Fjord; (2) Lake Åresjön $\left(63^{\circ} 23^{\prime} \mathrm{N}, 13^{\circ} 4^{\prime}\right.$ E), which is located in the Indalsälven river in central Sweden (Jämtland, Sweden), 167 and $284 \mathrm{~km}$ from Trondheim Fjord and Baltic sea coasts, respectively; (3) Lake Hasselasjön ( $\left.62^{\circ} 05^{\prime} \mathrm{N}, 16^{\circ} 48^{\prime} \mathrm{E}\right)$, which is close to the Swedish Baltic sea coast $(50 \mathrm{~km})$ in the Medelpad region. We selected low-productive lakes in each region in order to resemble marine oligotrophic conditions to the greatest possible extent. Lakes Jonsvatn and Åresjön are oligotrophic clearwater lakes, whereas lake Hasselasjön is slightly humic as the majority of lakes in this area (Supplementary Table 1). In addition, we collected water from one saline site, the Trondheim Fjord (salinity of $36,63^{\circ} 25^{\prime} \mathrm{N}, 10^{\circ} 23^{\prime} \mathrm{E}$ ) and one brackish site, the Baltic Sea coast (salinity of $4,62^{\circ} 01^{\prime} \mathrm{N}, 17^{\circ}$ 24' E). Supplementary Table 1 summarizes the main physicochemical characteristics at the different sampling sites. In situ temperature, conductivity, salinity and $\mathrm{pH}$ were measured using a Cond 3210 Set 2 conductivity meter (VWT, Weilheim, Germany) and a pH meter (Metroholm $826 \mathrm{pH}$ Mobile, Herisau, Switzerland), respectively. Total phosphorus, total nitrogen, dissolved organic carbon concentrations were determined as described in the Supplementary material (Supplementary Text 1).

Water samples (12 l from each lake) were pumped from $1 \mathrm{~m}$ below the surface into acid-washed $20 \mathrm{l}$ containers. Two sediments cores were taken per lake and the first $2 \mathrm{~cm}$ were sliced and kept cold and in darkness until further processing. In addition, samples of air bacteria in each lake area were taken.

Air sampling was done at each lake using a vacuum cleaner with a water tank (AquaStream DS 5600, 1400W, Kärcher, Winnenden, Germany). Before collection, lake water was autoclaved and the $\mathrm{pH}$ was reset to its initial value with the addition of $\mathrm{HCl}(1 \mathrm{~N})$. No more than $2 \mathrm{l}$ of the sterilized lake water was then

Table 1 Three-way analysis of variance testing for impact of salinity, source of inoculum and lake identity (and their interactions) on the proportion of freshwater and marine taxa as well as on niche breadth index along the marine gradient

Analysis of variance $\mathrm{F}$ values

\begin{tabular}{|c|c|c|c|c|c|c|c|}
\hline Tested-dependent variables & Salinity & Source & Lake ID & Sa:So & $S a: L a$ & So:La & Sa:So:La \\
\hline Percentage of freshwater OTUs & $15.6^{* * *}$ & $6^{* *}$ & $11.6^{* * *}$ & $3.8^{* * *}$ & 3 ** & $2.8^{*}$ & $2.9 * * *$ \\
\hline Percentage of marine OTUs & $23.1 * * *$ & NS & $7.9 * * *$ & $5.8^{* * *}$ & NS & NS & NS \\
\hline Niche breadth index & $166.2^{* * *}$ & $115.7^{* * *}$ & $16.2 * * *$ & $18.1 * * *$ & $5.9 * * *$ & $7.7 * * *$ & $7 * * *$ \\
\hline
\end{tabular}

Abbreviations: NS, not significant; OTU, operational taxonomic unit.

Sa, So and La refer to salinity, inoculum source and lake identity, respectively.

$P<0.0001^{* * *}, P<0.001^{* *}, P<0.01^{*}$. 
poured into the vacuum basket (previously ethanol cleaned) to reduce the dilution of the collected bacteria (Runar Thyrhaug, personal communication). Preliminary tests showed that the application of a vacuum with a flow rate of $3.3 \mathrm{~m}^{3}$ per minute for $3 \mathrm{~h}$ resulted in cells concentration that was sufficient for DNA extraction (data not shown). After sampling, collected air bacteria were kept in the dark and cold until the experiment started (up to 1 week later).

\section{Experimental set-up}

Water from all sites served as a source for both medium and inoculum. To prepare the medium, water was sequentially filtered through 142-mm AE glass fiber filters (Pall cooperation, East Hills, NY, USA) and autoclaved twice $\left(121^{\circ} \mathrm{C}, 20 \mathrm{~min}\right)$. $\mathrm{pH}$ was reset to in situ values between the two autoclaving cycles. The marine gradient was obtained by mixing sterile lake water and water from the Trondheim fjord (salinity 36) in different proportions (\% volume lake water-volume sea water): 100-0 (salinity 0), 75-25 (salinity 9), 50-50 (salinity 18), 25-75 (salinity 28), 0-100 (salinity 36). Later in the manuscript, the different treatments will be referred to by their corresponding salinities.

Three inoculum sources from each lake were used in the experiment: lake water, lake sediment and air. Before inoculation, all inocula were set to the same cell concentration $(1.4 \pm 0.6 \times$ $10^{6}$ cells $\mathrm{ml}^{-1}$ ). Lake inocula consisted of unfiltered lake water. Sediment inocula were prepared by diluting lake sediment 500 times in sterile lake water. Air bacteria were grown in sterile lake water until they reached the requested cell concentrations (up to 1 week). In total, $50 \mathrm{ml}$ of each inoculum was added to $450 \mathrm{ml}$ of sterile medium so that each lake, sediment and air assemblage were inoculated in its corresponding lake water mixed in different proportions with sterile sea water as previously described. Each treatment was replicated three times resulting in a total number of 156 cultures. In addition, marine controls were prepared by inoculating bacterial assemblages originating from the Trondheim Fjord and the Baltic Sea into their corresponding sterilized water. Other control cultures were autoclaved lake, Baltic and Norwegian sea water, which did not receive an inoculum. Supplementary Table 2 summarizes the design of the experimental set-up. The resulting batch cultures were incubated for 3 weeks in the dark at $18{ }^{\circ} \mathrm{C}$ corresponding to average temperatures measured in situ. Bacterial abundance was determined on days $0,3,7,10,13,17$, 19, 24, 26 using flow cytometry, whereas samples for bacterial community composition were taken at the end of the experiment only and analyzed using 454 pyrosequencing (see Supplementary Text 2).

\section{Pyrosequencing data processing}

From a total of 760325 reads, 465610 reads were assigned to the different treatments based on their barcodes. The removed sequences were ambiguous sequences, that is, reads with low quality as inferred from their flowgrams and those that did not carry the exact primer sequence. After implementation of these quality control criteria, PCR and pyrosequence noise filtering and chimera removal (Perseus) were carried out on the remaining reads using AmpliconNoise Version 1.24 (Quince et al., 2011). A total of 14482 reads was removed by Perseus using default settings (alpha $=-7.5$, beta $=0.5$ ) for falsepositive detection, with a resulting average number of 2573 sequences per sample (119-18295, minimum-maximum reads per sample, respectively). The 454 sequences have been deposited in the National Center for Biotechnology Information (NCBI) Short Read Archive under accession number SRA059612.

\section{Operational taxonomic unit assignments}

To assign reads into operational taxonomic units (OTUs), UCLUST was applied with a 99\% sequence similarity cutoff. The choice of this similarity cutoff instead of the usual $97 \%$ for bacteria was motivated by our need to use the finest possible resolution that would give us the highest certainty to detect typical marine taxa. A representative sequence from each of the resulting 11538 OTUs was classified using the naïve Bayesian classifier (Wang et al., 2007) implemented in QIIME (Caporaso et al., 2010) in combination with the provided rdp training dataset. The representative sequences of the 1470 most abundant OTUs from the subsampled dataset were chosen because they are likely to represent taxa that reactively grow in the cultures. They varied in relative abundance between $10 \%$ and $80 \%$, but collectively represent $90 \%$ of the total number of reads. They were then used in a local Basic Local Alignment Search Tool (BLAST)n search against the NCBI nr/nt database where the isolation source was extracted from the hits with $99 \%$ identity to the query sequence. After the environmental context information was extracted for each OTU, they were classified into environmental ontology bins represented by marine, freshwater and other habitats.

\section{Statistical analyses}

All statistical analyses were conducted using $\mathrm{R}$ (R Development Core Team, 2011; http://www. R-project.org/) and the vegan package. Permutational MANOVA (PERMANOVA, Anderson, 2001, www. stat.auckland.ac.nz/ mja/Programs.htm) was used to test for treatment (marine gradient), inoculum source and lake effects on community composition, based on Morisita-Horn dissimilarity among a set of 1931 OTUs that were represented by at least three reads. PERMANOVA was ran including the argument strata $=$ lake ID to account for differences among the three lakes. The Morisita-Horn metric was chosen because of its robustness against differing sample 
sizes (Wolda, 1981, Krebs, 1999). Non-metric multidimensional-scaling plots of a Morisita-Horn distance matrix were used to visualize differences in community structure ( $\beta$-diversity) between cultures where freshwater and air assemblages were regrown under marine conditions and where Baltic and Norwegian Sea bacterial communities were inoculated in their own water.

To address whether there were changes in the proportion of taxa with a wide niche breadth, that is, taxa that can tolerate a wide range of salinities, along the marine gradient we used an OTU scoring system based on the presence of OTUs at the different levels of the marine gradient. For this, the subsampled OTU table was transposed into a presence-absence matrix to obtain an 'OTU score' bounding between 0 (OTU not detected) and 5 (OTU present along the whole gradient) and which was obtained by summing the 0 and 1 for each OTU along the marine gradient for each location and inoculum source. To estimate how OTU scores vary along the marine gradient and differ between the different inoculum sources, we modified the presence-absence matrix by substituting the ' 1 ' with the actual OTU score. The total niche breadth score for each sample, that is, each inoculum source at each salinity, was then estimated by averaging the scores of OTUs that composed the community and corrected for the total number of OTUs detected in the sample. A high average OTUs score indicates a large proportion of OTUs with wide niche breadth, that is, habitat generalists, is present in the community, that is, in our case an increasing proportion of taxa that can cope with a wide range of salinities. On contrary, a low average OTU score indicates a larger proportion of habitat specialists with a narrow niche width, that is, in our case taxa that are restricted to specific salinity levels.

Three-way analysis of variance analyses were used to test for a significant effect of salinity, inoculum source, lake identity as well as their interactions on both the proportion of habitats generalists (average niche breadth scores) and the percentage of marine taxa and freshwater taxa along the marine gradient, respectively. Post hoc Tukey-HSD tests were further used to determine the levels at which significant differences were detected. Adjustments for multiple comparisons were applied using the Bonferroni correction procedure. The values for percentages of marine and freshwater taxa were arcsine square roottransformed and OTUs niche breadth scores were log-transformed to approach normal distribution.

\section{Results}

Bacteria followed typical batch culture growth curves consisting of an initial lag phase (from day 0 to 3), an exponential growth phase (from day 3 to 19) and a stationary phase (starting on day 19) in the majority of the incubations (Supplementary Figure 1). Kruskal-Wallis tests showed an overall significant difference in growth rates between the three inoculum sources, with the lowest growth rates in air cultures $\left(\chi^{22}=8.43\right.$, d.f. $\left.=2, P=0.015\right)$. There were no significant differences between the three sources at freshwater conditions as well as at conditions where fresh and marine waters were mixed at a ratio of 75:25 (v:v). In contrast, bacterial communities in both lake and sediment cultures had higher growth rates than those inoculated with air bacteria when lake water and marine water were mixed at proportions of 50:50 $\left(\chi^{2}=11.65\right.$, d.f. $=2$, $P=0.003)$ and $25: 75\left(\chi^{2}=15.69\right.$, d.f. $\left.=2, P=0.0004\right)$. Under marine conditions, sediment cultures had the highest growth rates $\left(\chi^{2}=12.12\right.$, d.f. $\left.=2, P=0.002\right)$.

The results from the PERMANOVA analyzes showed a significant effect of marine conditions $\left(F=11.5, \quad r^{2}=0.07 \quad P<0.001\right), \quad$ inoculum source $\left(F=7.3, r^{2}=0.09, P<0.001\right)$ as well as their interaction $\left(F=4, \quad r^{2}=0.05, \quad P<0.001\right)$ on community composition. Non-metric multidimensional scaling showed that although exposed to similar marine conditions, freshwater and air communities remained distinct from marine bacteria inoculated into their original water, even at the highest salinity (Supplementary Figure 2). The taxa (OTUs) retrieved in the different cultures were scored based on their assignments to either the freshwater or marine biome, when screening closely related sequences in the NCBI database. One taxon appeared to be highly abundant in all cultures and was identified as Acinetobacter from the Gammaproteobacteria. In addition, lake cultures had high abundances of Betaproteobacteria (Limnobacter) and Actinobacteria (Rhodococcus). Sediment cultures were dominated by Bacteroidetes, Actinobacteria and Gammaproteobacteria.

Both, marine conditions and inoculum source had significant effects on the percentage of freshwater taxa (analysis of variance Table 1), which was highest at freshwater salinities and in lake and sediment cultures compared with higher salinities and air cultures, respectively (Figure 1, Supplementary Table 3).

Marine conditions and lake identity but not inoculum source had a significant effect on the percentage of marine taxa (Table 1). Post hoc tests showed that communities originating from Jonsvatn contained a higher proportion of marine taxa than the inland lake (Åresjön) and to a weaker extent, the lake close to the Baltic Sea $(P=0.1$, Hasselasjön) at higher salinities (Figure 2b, Supplementary Table 3). Post hoc tests showed that lake and sediment cultures had a higher percentage of marine taxa at higher salinities compared with air cultures (Figure 2).

The average OTU scores and thereby the proportion of taxa with wide niche breadths, increased along the marine gradient (Figure 3, Supplementary Table 3). The average niche breadth of taxa was significantly influenced by inoculum source $(F=115.6, P<0.0001)$ with sediment communities 
a

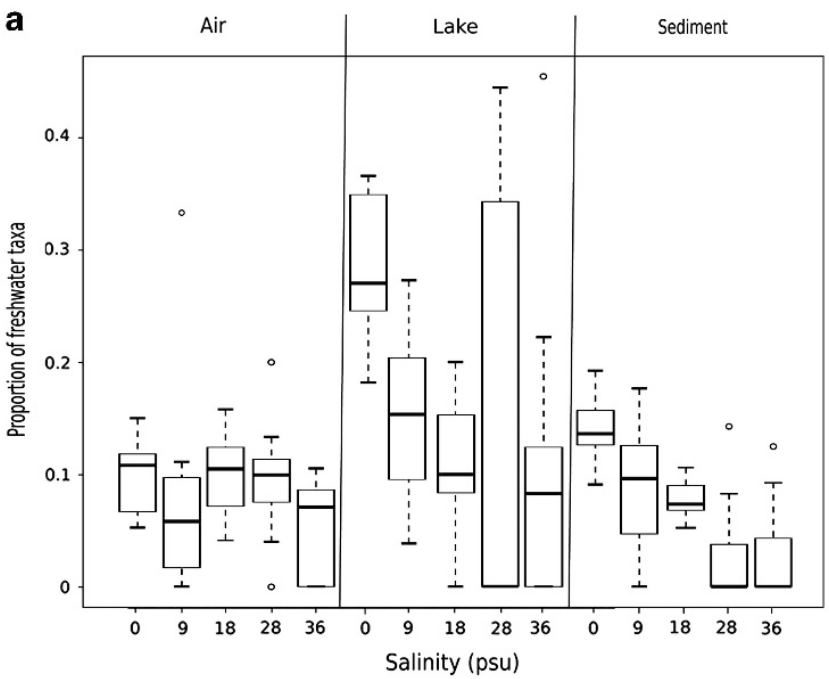

b

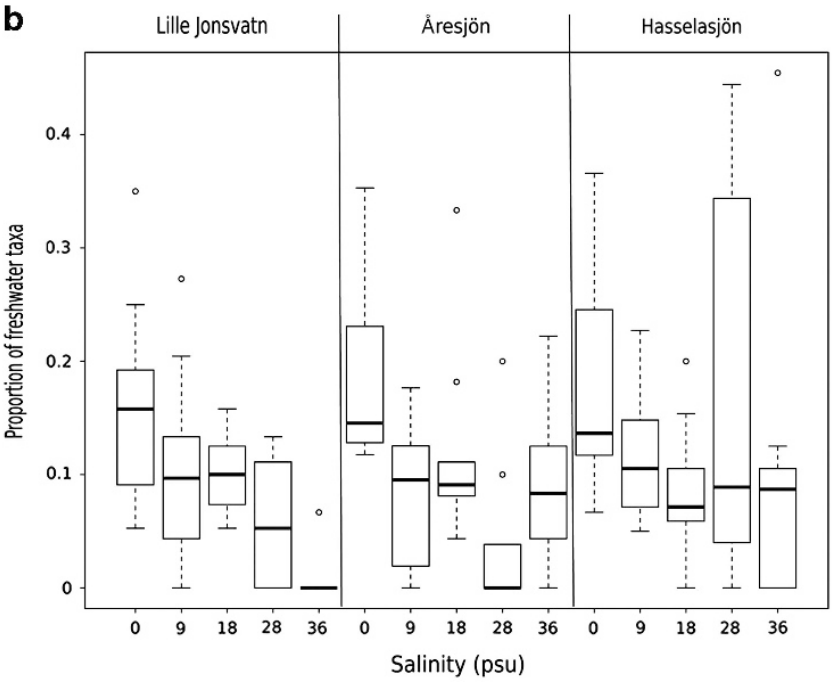

Figure 1 Contribution of freshwater taxa along the marine gradient for the different inoculum sources (a) and the different lakes sampled (b). The contribution of freshwater taxa was defined by the percentage of reads associated with freshwater OTUs as determined by homology searches against NCBI.
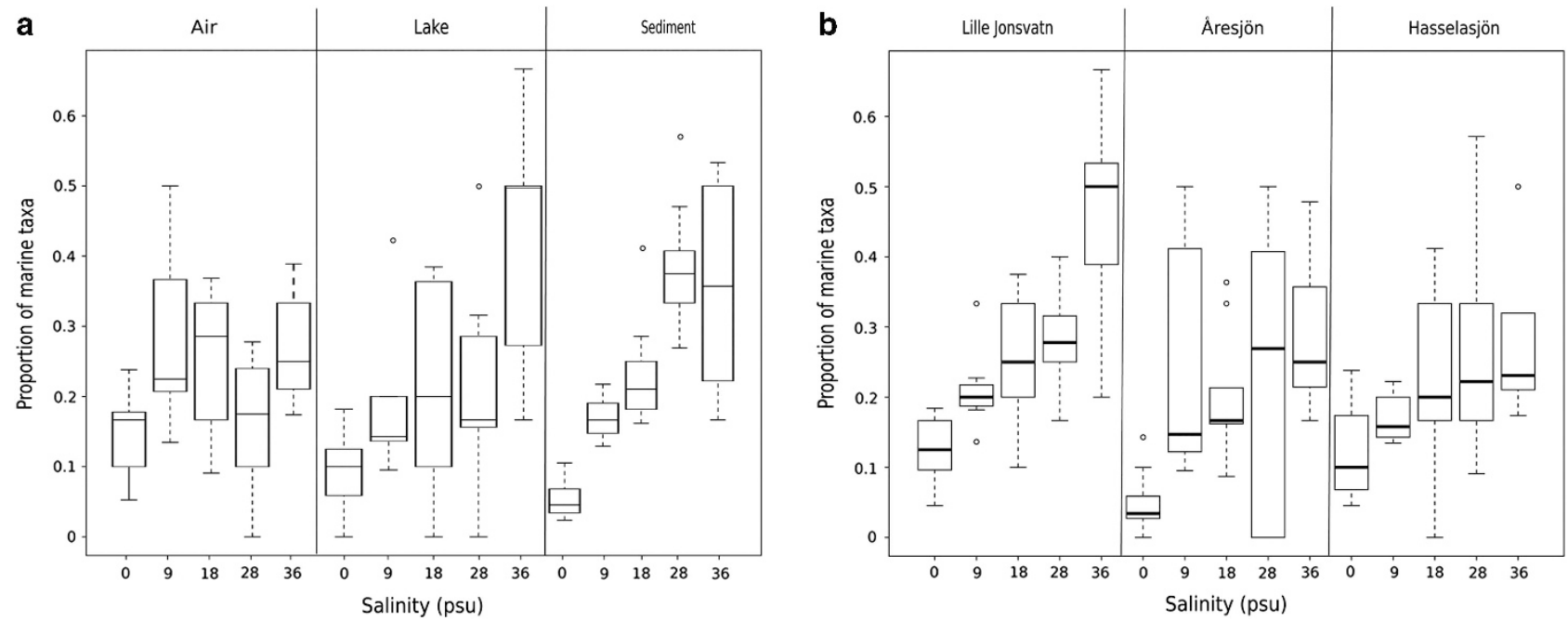

Figure 2 Contribution of marine taxa along the marine gradient for the different inoculum sources (a) and the different lakes sampled (b). The contribution of marine taxa was defined by the percentage of reads associated with marine OTUs as determined by homology searches against NCBI.

presenting the lowest scores and air the highest (Figure 3a). Salinity $(F=166.2, P<0.0001)$. Lake identity $(F=16.2, P<0.0001)$ had also an effect on the average niche breadth of taxa retrieved in the cultures (Figure $3 \mathrm{~b}$ ) and all two-way and the threeway interaction terms were significant as well (Supplementary Table 3).

\section{Discussion}

The main aim of this study was to test how bacterial communities in lake water, lake sediments and air respond to an increase in the exposure to marine conditions. Our first hypothesis that marine taxa can be retrieved after the exposure to marine conditions was supported by our results. On the contrary, we have to reject our second hypothesis that marine bacteria are mainly present in sediments, since we found similar levels in the percentage of marine taxa for both sediment and lake bacteria. Our results were in agreement with the idea that the proportion of marine taxa was generally higher in the lake close to the Norwegian Sea compared with the other lakes. Finally, our results also suggest that recruitment of habitat generalists, that is, taxa with a wide niche breadth (in our case taxa found at several of the salinity levels), was an important mechanism by which freshwater communities responded to increases in salinity along the marine gradient. Moreover, there were differences among the source communities since the increase in generalists was 

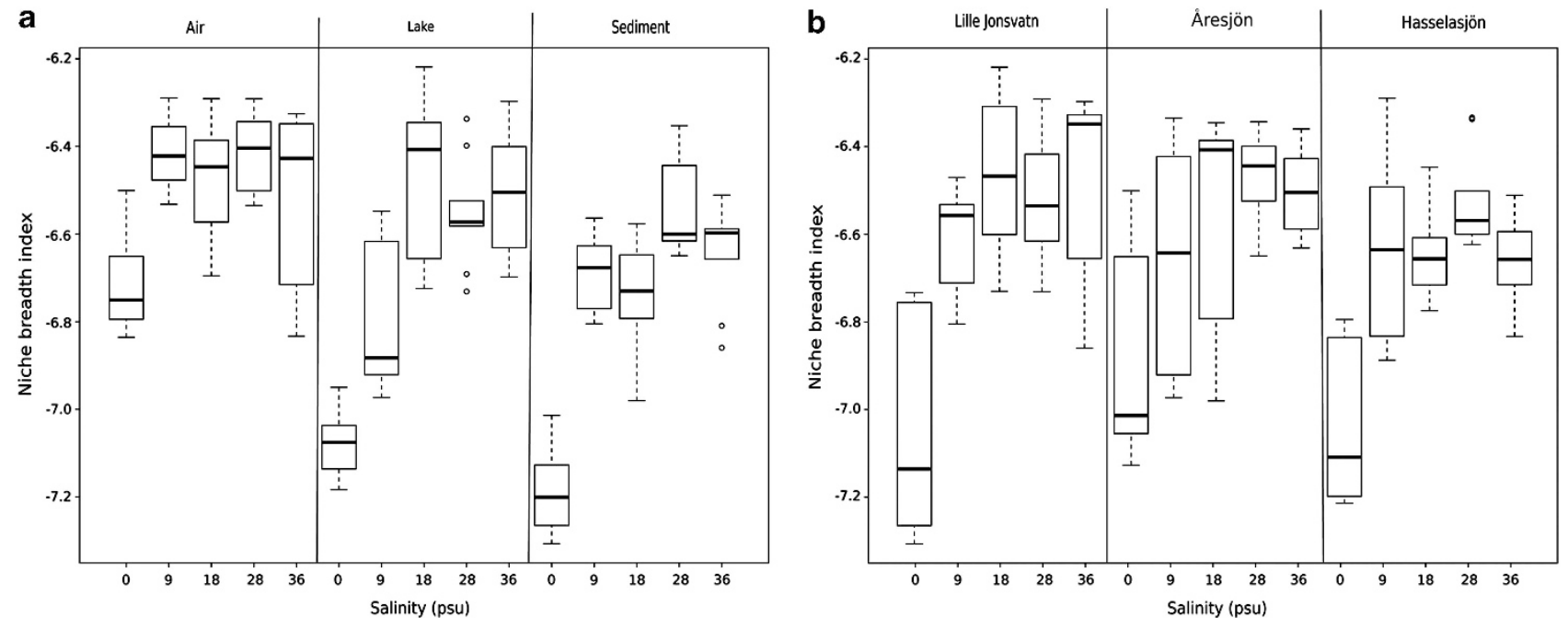

Figure 3 Changes in average niche breadth scores of OTUs along the marine gradient for each source of inoculum (a) as well as each lake sampled (b). High values mean that OTUs remain abundant along the marine gradient, that is, that they are considered to be habitat generalists.

highest in the case of sediment and lakes cultures, while air communities initially contained a significantly larger proportion of habitat generalists than the other sources.

Generally, our results support previous suggestions that marine taxa can accumulate over time in for example, sediments (for example, Davies et al., 1995) and that bacterial communities contain a large reservoir of rare or dormant taxa that can be regrown under suitable conditions (for example, Hubert et al., 2009; Jones and Lennon, 2010; Caporaso et al., 2012; Sjöstedt et al., 2012). These can potentially play an important role by influencing the response of bacterial communities to environmental change and disturbances (Shade et al., 2012). Contrary to our expectations, however, marine bacteria were retrieved to the same extent from lake water and sediments. Moreover, we could also retrieve typical marine bacteria from the air samples, suggesting that air might contribute to the build-up of marine representatives in the other two sources, possibly after air deposition events like previously described (for example, Aller et al., 2005). However, air communities showed generally a deviating pattern, since the percentage of marine OTUs did not increase as strongly in response to higher salinities as it did for lake water and sediment. We argue that this pattern is sustained by the overall higher proportion of taxa with a wide niche breadth, that is, habitat generalists, compared with the other two inoculum sources. This fits with the idea that air bacteria are composed of a diverse range of taxa from different sources that are able to cope with a wide range of environmental conditions (Fahlgren et al., 2010) and consequently change less when transferred to novel environmental conditions.

As expected we found that communities originating from the lake closest to the sea harbored more marine taxa compared with lakes further away, that is, we found generally a higher proportion of marine taxa in cultures with inoculum from lake Lille Jonsvatn, which is located $\sim 20 \mathrm{~km}$ away from the coast of the Norwegian sea. This result indicates that dispersal events that transported marine taxa from the sea were more frequent to the lake in closer proximity to the sea compared with the other two lakes, which were either located in the mountains or close to the Swedish Baltic Sea coast, which is a brackish environment. An alternative explanation to our result is that lake Lille Jonsvatn was previously below sea level (Reite, 1983; Hedenström and Risberg, 1999); that is, before ancient displacement of the Norwegian seashore line ( $\sim 8000$ years ago), and marine bacteria may have remained in the lake since then. This explanation, however, seem unlikely, especially for the sediments, since we only used surface sediments which were much more recently formed.

Concerns can always be raised about experimental systems as ours being artificial, for instance, by selecting only for fast-growing opportunists (for example, Eilers et al., 2000; Fuchs et al., 2000) and for not reflecting the entire community. This phenomenon was seen in our systems due to the high abundance of Gammaproteobacteria, which are generally known to be fast-growing opportunists (Pinhassi and Berman, 2003; Newton et al., 2011). Thus, community composition in our cultures certainly differed from natural environments as ubiquitous and abundant marine bacteria such as SAR 11 (for example, Morris et al., 2002) were not found in any of our cultures. However, cultures receiving marine inocula were dominated by taxa reported from other marine ecosystems (for example, Kirchman et al., 2010) and were very different from our cultures of freshwater inocula even under marine conditions (Supplementary Figure 2). This shows that our experimental systems did not show a 
general bias against marine bacteria per se. The proportion of OTUs assigned as marine was high in the cultures of highest salinity (up to about $50 \%$, Figure 3). Except under freshwater conditions, freshwater OTUs were less common than marine OTUs in the cultures. Still, the relative numbers have to be interpreted with caution as our habitat annotations rely on homology searches against sequences present in public databases as well as the source entries that are available from them. As marine entries are highly over-represented compared with freshwater entries, this leads to a higher proportion of marine signal in the habitat annotations. In addition, only $50 \%$ of the sequences could be annotated to a habitat when using a $99 \%$ similarity cutoff pinpointing to a low coverage of similar sequences in the NCBI database. Still, a high similarity level for defining an OTU and for assigning OTUs to either marine or freshwater is necessary to minimize the chances to lump typical marine and freshwater sequences together into a single OTU. The effect of sequence resolution on microbes' ecology and responses to environmental changes were also recently highlighted (for example, Youngblut et al., 2013). Hence, the ratio between marine and freshwater taxa presented in this study needs to be treated with caution; nevertheless, the trends that we found in terms of proportional changes in response to increasing marine conditions should hold.

To conclude, we have shown that the exposure of lake, sediment and atmospheric bacterial communities to marine conditions led to an increase in the proportion of marine taxa and habitat generalists, but a decrease of freshwater taxa, and that this response was stronger in lake and sediment compared with air communities. This suggests that marine taxa can readily grow from freshwater sources, but that the response was likely driven by the growth of habitat generalists that are found in marine systems. More studies are now needed to test whether a similar response is also found in situations where natural communities from different sources are exposed to marine conditions, as well as other disturbances, such as acidification, eutrophication, brownification and moderately changed temperature.

\section{Conflict of Interest}

The authors declare no conflict of interest.

\section{Acknowledgements}

We thank members from Sealab (NTNU, Trondheim, Norway) for their help for sampling and providing nutrient data of Trondheim fjord and more especially Helge Reinertsen who further provided access and facilities to lake Jonsvatn. We are also grateful to Runar Thyrhaug (U. Bergen, Norway) for advices on air sampling, as well as Mercè Berga, Örjan Östman, Richard Svanbäck and Peter Eklöv for discussions concerning statistics, Jan Johansson for assistance in bacterial enumeration in sediments and anonymous reviewers for their valuable comments on previous versions of the manuscript. We also want to thank the Uppsala Multidisciplinary Center for Advanced Computational Science (UPPMAX) for providing computational resources for data storage and analysis. Financial support was provided by Olsson-Borghs foundation and FORMAS to SL, WennerGren foundations to ESL and JC, the Swedish research council to ESL, and the Swedish Foundation for strategic research to $\mathrm{AE}$.

\section{References}

Aller JY, Kuznetsova MR, Jahns CJ, Kemp PF. (2005). The sea surface microlayer as a source of viral and bacterial enrichment in marine aerosols. J Aerosol Sci 36: 801-812.

Anderson MJ. (2001). Permutation tests for univariate or multivariate analysis of variance and regression. Can J Fish Aquat Sci 58: 626-639.

Caporaso JG, Kuczynski J, Stombaugh J, Bittinger K, Bushman FD, Costello EK et al. (2010). QIIME allows analysis of high-throughput community sequencing data. Nat Methods 7: 335-336.

Caporaso JG, Paszkiewicz K, Field D, Knight R, Gilbert JA. (2012). The Western English Channel contains a persistent microbial seed bank. The ISME J 6: 1089-1093.

Davies CM, Long JA, Donald M, Ashbolt NJ. (1995). Survival of fecal microorganisms in marine and freshwater sediments. Appl Environ Microbiol 61: 1888-1896.

Declerck SAJ, Winter C, Shurin JB, Suttle CA, Matthews B. (2013). Effects of patch connectivity and heterogeneity on metacommunity structure of planktonic bacteria and viruses. ISME J 7: 533-542.

Eilers H, Pernthaler J, Amann R. (2000). Succession of pelagic marine bacteria during enrichment: a close look at cultivation-induced shifts. Appl Environ Microbiol 66: 4634-4640.

Fahlgren C, Hagström Å, Nilsson D, Zweifel UL. (2010). Annual variations in the diversity, viability, and origin of airborne bacteria. Appl Environ Microbiol 76: 3015-3025.

Finlay BJ, Maberly SC, Cooper JI. (1997). Microbial diversity and ecosystem function. Oikos 80: 209-213.

Fuchs BM, Zubkov MV, Sahm K, Burkill PH, Amann R. (2000). Changes in community composition during dilution cultures of marine bacterioplankton as assessed by flow cytometry and molecular biology techniques. Environ Microbiol 2: 191-201.

Gibbons SM, Caporaso JG, Pirrung M, Fielde D, Knight R, Gilbert JA. (2013). Evidence for a persistent microbial seed bank throughout the global ocean. Proc Natl Acad Sci USA 10: 4651-4655.

Hanson CA, Fuhrman JA, Horner-Devine MC, Martiny JBH. (2012). Beyond biogeographic patterns: processes shaping the microbial landscape. Nat Rev Microbiol 10: $497-506$.

Hedenström A, Risberg J. (1999). Early Holocene shoredisplacement in southern central Sweden as recorded in elevated isolated basins. Boreas 28: 490-504.

Hubert C, Loy A, Nickel M, Arnosti C, Baranyi C, Brüchert V et al. (2009). A constant flux of diverse thermophilic bacteria into the cold Arctic seabed. Science 325: 1541-1544. 
Jones SE, Newton R, McMahon KD. (2008). Potential for atmospheric deposition of bacteria to influence bacterioplankton communities. FEMS Microbiol Ecol 64: 388-394.

Jones SE, Lennon JT. (2010). Dormancy contributes to the maintenance of microbial diversity. Proc Natl Acad Sci USA 107: 5881-5886.

Kirchman DL, Cottrell MT, Lovejoy C. (2010). The structure of bacterial communities in the western Arctic Ocean as revealed by pyrosequencing of $16 \mathrm{~S}$ rRNA genes. Environ Microbiol 12: 1132-1143.

Krebs CJ. (1999). Ecological Methodology, 2nd edition Benjamin/Cummings: Menlo Park, California.

Lindström ES, Langenheder S. (2012). Local and regional factors influencing bacterial community assembly. Environ Microbiol Reports 4: 1-9.

Logares R, Bråte J, Bertilsson S, Clasen JL, Shalchian-Tabrizi K, Rengefors K. (2009). Infrequent marine-freshwater transitions in the microbial world. Trends Microbiol 17: 414-422.

Lozupone CA, Knight R. (2007). Global patterns in bacterial diversity. Proc Natl Acad Sci USA 104: 11436-11440.

Morris RM, Rappé MS, Connon SA, Vergin KL, Siebold WA, Carlson CA et al. (2002). SAR11 clade dominates ocean surface bacterioplankton communities. Nature 420: $806-810$.

Newton RJ, Jones SE, Eiler A, McMahon KD, Bertilsson S. (2011). A Guide to the natural history of freshwater lake bacteria. Microbiol Mol Biol Rev 75: 14-49.

Pinhasi J, Berman T. (2003). Differential growth responses of colony-forming a- and g-Proteobacteria in dilution culture and nutrient addition experiments from lake Kinneret (Israel), the eastern
Mediterranean Sea, and the gulf of Eilat. Appl Environ Microbiol 69: 199-211.

Quince C, Lanzen A, Davenport RJ, Turnbaugh PJ. (2011). Removing Noise from pyrosequenced amplicons. BMC Bioinfo 12: 38.

Reite AJ. (1983). Trondheim. Description of the quatemary geological map 1621 IV (scale 1:50000). Nor Geol Unders 391: 1-44.

Shade A, Read JS, Youngblut ND, Fierer N, Knight R, Kratz TK et al. (2012). Lake microbial communities are resilient after a whole-ecosystem disturbance. ISME J 6: 2153-2167.

Sjöstedt J, Koch-Schmidt P, Pontarp M, Canbäck B, Tunlid A, Lundberg P et al. (2012). Recruitment of members from the rare biosphere of marine bacterioplankton communities after an environmental disturbance. Appl Environ Microbiol 78: 1361-1369.

Székely AJ, Berga M, Langenheder S. (2013). Mechanisms determining the fate of dispersed bacterial communities in new environments. ISME J 7: 61-71.

Tamames J, Abellán JJ, Pignatelli M, Camacho A, Moya A. (2010). Environmental distribution of prokaryotic taxa. BMC Microbiol 10: 85

Wang Q, Garrity GM, Tiedje JM, Cole JR. (2007). Naïve Bayesian classifier for rapid assignment of rRNA sequences into the new bacterial taxonomy. Appl Environ Microbiol 73: 5261-5267.

Wolda H. (1981). Similarity indices, sample size and diversity. Oecologia 50: 296-302.

Youngblut ND, Shade A, Read JS, McMahon KD, Whitaker RJ. (2013). Lineage-specific responses of microbial communities to environmental change. Appl Environ Microbiol 79: 39-47.

Supplementary Information accompanies this paper on The ISME Journal website (http://www.nature.com/ismej) 